\title{
Complexity Induced Lifshitz Ordering with Multifractal Antiscreening/Screening (CILOMAS)
}

\section{Tom T.S. Chang}

Kavli Institute for Astrophysics and Space Research

Massachusetts Institute of Technology, Cambridge, MA 02139, USA

\begin{abstract}
We demonstrate that renormalization-group effects of scale-running propagatorcoupling constants due to classical fluctuations can induce antiscreening/screening and multifractal symmetry breakings among various helical and other ordered states of generalized Lifshitz character leading to novel phase-transition associated complexities in condensed matter physics and gravitational evolution. Such phenomenon can exhibit the sporadic and localized appearance of virtual particles and in the context of cosmological evolution, the coarse-grained scale-running of the gravitational constant $G$ due to classical fluctuations may provide a partial explanation to the dark matter mystery.
\end{abstract}

Keywords: Renormalization group, Complexity, Lifshitz character, Antiscreening and screening, Virtual Particles, Dark matter

\section{Introduction}

In standard renormalization-group analyses, the coupling constant associated with the propagator is generally assumed to be scale-independent and conveniently normalized to unity. When such coupling constants are running (i.e., scale-dependent), the 
propagators will allow ordered states in condensed matter physics and dynamical systems to take on complex helical characteristics. This property can introduce spatiotemporal localized symmetry breakings among states of helical and uniform/commensurate orders (Lifshitz behavior [1.2]) leadings to multifractal complexity with antiscreening/screening effects. We demonstrate in this letter that such phenomenon can provide the explanation for the appearance of virtual particles in static and dynamical systems. In the context of cosmology, it may provide a possible partial explanation for the apparent observations of dark matters.

\section{The Exact Renormalization Group (RG)}

Our starting point is the exact, non-perturbative, one-particle-irreducible RG formulation introduced by Nicoll and Chang [3] for static critical phenomena and Chang et al. [4] for dynamical systems far from equilibrium, based on the Wilsonian viewpoint [5-7]. We are interested in understanding the evolution of a complex system by viewing its coarse-grained prescription including classical fluctuations at larger and larger scales. To obtain a RG generator for such a process, we derive a differential equation for the scale-dependent effective action $A\left(\psi_{k}, \psi_{q}, \bar{\psi}_{p}\right)$ defined in analogy to the effective action (Helmholtz free energy) $\bar{A}(\bar{\psi})$, the generating functional for the one-particle irreducible n-point correlation functions of a fluctuating field (order parameter) $\psi$, which can be a scalar, vector or tensor with or without time dependence where $\bar{\psi}_{k} \equiv\left\langle\psi_{k}\right\rangle$. We accomplish this by saddle point functional integrations of $A\left(\psi_{k}\right)$ over $\psi_{k}$ fluctuations within infinitesimal momentum (k)-space shells successively inward, where q represents 
the present momentum shell of integration and $\mathbf{p}$ the shells of prior integrations (with $\bar{\psi}_{p}^{\prime}=\overline{\psi_{p}}\left(\psi_{k}, \bar{\psi}_{q}\right)$ evaluated at the $q$ shell saddle point). The result is [3]:

$$
\frac{\partial A}{\partial \ell}=\frac{1}{2} \operatorname{tr} \ln \left(A_{q q^{\prime}}-A_{q p}\left(A^{-1}\right)_{p p^{\prime}} A_{p^{\prime} q}\right)
$$

(with the indices of the matrix $A$ suppressed) where $\ell$ is the shell scaling (RG) parameter, $A_{q p}=\delta^{2} A /\left.\delta \psi_{q} \delta \bar{\psi}_{p}\right|_{\left\{\psi_{q}\right\}=\left\{\bar{\psi}_{q}\right\}}$ and analogous expressions for the other Hessians, $A^{-1}$ is the inverse of $A$, and the trace (tr) includes integrations over continuous indices.

For (1), the momentum shells are required to only collectively span the range of k . For isotropic shells, the generator may be conveniently written as [8]:

$$
\frac{\partial A}{\partial \ell}=\frac{1}{2} \int \frac{d \Omega}{(2 \pi)^{d}} \operatorname{tr} \ln \left(A_{q,-q}-\int \frac{d p}{(2 \pi)^{d}} \int \frac{d p^{\prime}}{(2 \pi)^{d}} A_{q p}\left(A^{-1}\right)_{p p^{\prime}} A_{p^{\prime},-q}\right)
$$

where $d \Omega$ is the differential element of the surface defined by $|\mathbf{q}|=1$.

The differential RG functional generator is exact, non-perturbative, and in closed form. Being formulated in terms of the one-particle irreducible scale-dependent effective action, it differs from and is more compact than that of the Wegner-Houghton generator $[7,9]$.

\section{Complexity}

Complexity phenomenon can set in from an interacting system composed of many individual elements. If the interactions are nonlinear and long-ranged, quite often large scale structures are generated - each (well developed or partially formed) structure being composed of many individual elements behaving more or less coherently together. These "coherent structures" (such as the complex tangled helically ordered dynamical states to be described in this letter) can have varied shapes and sizes. And the ensuing behavior 
resulting from the diversified interactions of these structures can become very complicated [10]. RG methods based on the Wilsonian viewpoint as described in Section II are designed to handle such physical complications involving many scales [6].

\section{Application}

We shall now discuss the phenomenon of Complexity Induced Lifshitz Ordering with Multifractal Antiscreening/Screening (CILOMAS). Consider, as an example, the coupling of the running of the gravitational constant in dynamical evolution. At small scales, multifractal structures related to the nonlinear evolution of coupling constants in RG can set in and these fluctuations are akin to those commonly observed in hydrodynamic turbulence $[11,12]$. Such phenomena do not need the influence or existence of nearby fixed points in RG phase space.

At larger scales, instabilities and self-organizations set in due to localized gravitational collapses and condensations, as well as other static or dynamical effects. These events can exhibit bona fide symmetry-breaking phenomena with associated fixed points similar to those commonly observed in condensed matter physics. We are mainly interested in the gravitational evolution during the matter-dominated era within the domain of non-relativistic dynamic motion. Thus, we shall start with the Einstein-Hilbert effective action and work under the Newtonian approximation by assuming (i) the gravitational field is weak, (ii) the variations of fields are slow in time, and (iii) the particles are nonrelativistic. Therefore, the only surviving metric element is $g^{00} \approx 1-h_{00}$ with $\left|h_{00}<<1\right|$. The approximate effective action is then [13]:

$$
A=\int(8 \pi G)^{-1}(\nabla \varphi)^{2} d^{3} x d t-\sum m \int \varphi d t+\sum(m / 2) \int v^{2} d t
$$


where the gravitational potential $\varphi=c^{2} h_{00} / 2, G$ is the gravitational constant, and $(m, v)$ are the masses and corresponding velocities. We do not include the cosmological constant because the running of it will have only minor feedback to the scaling of $G$. To (3), we can append other allowable higher order interaction terms and the corresponding coupling constants due to fluctuations through coarse-graining, source terms, stochasticity, and the RG transformation.

Consider the situation of symmetry breakings due to the appearance of fixed points in the ordering of the gravitational potential $\varphi$ under the influence of the scalerunning of the gravitational constant. This is the most important ordering and symmetrybreaking effect in gravitational evolution as can be seen from (3). Even with $G$ as an irrelevant parameter, the running of its $\mathbf{k}$-dependence nevertheless has a direct influence on the $\varphi$-ordering due to the fluctuations as it has a direct effect on the characteristics of the propagator. Eventually $G$ may develop special cusp characteristics (due to linear or nonlinear interaction effects and instabilities) at nontrivial values of $G^{*}$ 's with enhanced singular scaling characteristics with respect to $\mathbf{k}$. At these cusp singularity-induced fixed points (where, e.g., $G-G^{*} \sim \exp \left(\lambda_{G} \ell\right) \sim\left|k-k^{*}\right|^{\lambda_{G}}$ where $\lambda_{G}$ may be an irrational number for the isotropic essentially diagonalized case), higher order symmetry breakings among helically and uniformly or commensurately ordered states may set in [1,2]. The helical orderings can have sinusoidal variations of arbitrary periodicity in different directions, forming complex tangled coherent structures similar to those observed in the cosmic web.

Under either of the above scenario, the critical propagator may take on the generic form of the generalized Lifshitz character [2], $\sum_{i=1}^{J}\left|\mathbf{k}_{i}\right|^{\sigma_{i}}$, where each $\mathbf{k}_{i}$ is a $d_{i}$ - 
dimensional vector and $\sigma_{i}$ are the corresponding propagator exponents, differentiating the phases among the helically ordered states from the various uniformly or commensurately ordered states or among the different anisotropic helical states, In addition, of course, it also allows for the phase transitions among various orders of uniform/commensurate states.

To demonstrate the possibility of the existence of such symmetry breakings, we search for generalized perturbative fixed points based on the differential RG generator (1). Generalized perturbative fixed points were first calculated by Nicoll et al. [14] for the isotropic $k^{2}$-propagator by supposing the competing phases are spatially uniform and thus to lowest order the correlation length exponent (anomalous dimension) $\eta=0$. Under such an assumption which may be verified a posteriori, the RG generator of (2) becomes a nonlinear partial differential equation. It has a trivial, "i.e., Gaussian", fixed point at $A^{*}=0$. Linearizing the equation around the Gaussian fixed point and solving, one obtains a set of Gaussian eigenfunctions which are related to the associated Laguerre polynomials with eigenvalues $\lambda_{p}=d+p(2-d)$, where $d$ is the spatial dimension and $p$ the order of the polynomial expansion of the $\varphi$-coupling. To locate a fixed point of small perturbation, we may choose any one of the admissible eigenvalues to be small, e.g., $\varepsilon_{O} \equiv d+O(2-d)<<1$, and search for a perturbed fixed point of the same order of magnitude. This can be accomplished by setting $A_{O}^{*}=a_{O} Q_{O} \varepsilon_{O}$ where $Q_{O}$ is the Oth order eigenfunction. With $\partial A / \partial \ell=0$, the linear and quadratic terms in $A$ of the right hand side of the RG equation (expanded in terms of the Gaussian eigen-polynomials) may be balanced to second order in $\varepsilon_{O}$ by the appropriate choice of the value of $a_{O}$. 
The evaluation of $a_{O}$ and eigenvalues of the RG equation linearized with respect to the perturbed fixed point may be accomplished utilizing the orthogonal conditions of the associated Laguerre polynomials in terms of inner products via standard applied mathematics techniques.

This procedure may easily be generalized to the search for the perturbed nontrivial generalized Lifshitz fixed points. To accomplish this, we now analyze the partial differential equation approximation of the RG generator (1) which is valid for anisotropic momentum shells. With the appropriate differential scale changes dictated by the anisotropic property of the effective action involving the critical Lifshitz propagator exponents $\sigma_{i}$ for each of the corresponding $d_{i}$-dimensions we can easily calculate the perturbed fixed points and their associated eigenvalues. Such algebraic manipulations were reported in the original paper that introduced the generalized Lifshitz phenomenon [2]. Details follow step-by-step the calculations outlined in Eqs. $(4.10-4.20)$ of [8] and the results were given explicitly in [2]. For the scalar order parameter $\varphi$, the gravitational potential, we obtain the expression for the perturbed eigenvalues to first order in $\varepsilon_{O} \equiv \lambda_{O}$ for $d$ less than the borderline dimension $d_{b}$ determined by $\sum_{i=1}^{J} d_{i} / \sigma_{i}=O /(O-1)$, where $O$ is the order of phase transition.

$$
\lambda_{p}^{\prime}=\lambda_{p}-2 \varepsilon_{O}\langle O, p ; p\rangle /\langle O, O, O\rangle
$$

with $\lambda_{p} \equiv\left[\sum_{i=1}^{J} d_{i} \sigma_{>} / \sigma_{i}\right](1-p)+p \sigma_{>}$being the Gaussian eigenvalues. Here $\sigma_{>}$is the largest propagator exponent, and the inner products $\langle O, p ; p\rangle$ of the Gaussian eigenoperators are expressible in terms of the binomial coefficients in closed form 


$$
\langle O, p ; p\rangle=\sum_{j=0}^{[O / 2]}\left(\begin{array}{l}
p \\
j
\end{array}\right)\left(\begin{array}{c}
p-1 / 2 \\
j
\end{array}\right)\left(\begin{array}{c}
2 p-2 j \\
O-2 j
\end{array}\right)
$$

These fixed points are located at $H_{O}^{*} \sim \varepsilon_{O} Q_{O}$ where $Q_{O}$ is the corresponding Gaussian eigenfunction. They contain all the generalized Lifshitz-character perturbative fixed points involving various helical orderings of $\varphi$ as well as the non-Lifshitz fixed points [14], many of which are physically realizable. From $(4,5)$, all scaling exponents of the coupling constants involving $G$ and $\varphi$ may be calculated.

The coarse-grained scaling property of the cusp-like (possibly) singular variations of the gravitational constant $G$ in $\mathbf{k}$ near $G^{*}$ 's will provide an apparent coarse-grained modification of the $(1 / r)$-property of the gravitational potential and thereby induce the apparent anomalous increasing (due to the increasing of $G-G^{*}$ or the decreasing of $\mid k-k *)$ and decreasing trends of the antiscreening/screening effect that may be interpreted as the appearance of virtual particles. Such apparent anomalies are the direct manifestation of the Lifshitz dimensions $\sigma_{i}$ of the critical propagator and antiscreening may be interpreted as dark matters in coarse-grained observations of the cosmic web at large scales.

In addition to the ordering effects of $G$ related to $\varphi$ alone, there can be competing orderings with the other field variables such as $m$ and $\mathbf{v}$ and accompanying higher order interaction terms. These symmetry-breaking effects can introduce further sporadic and localized (both fading-in and fading-out) antiscreening/screening effects on $G$.

\section{Spatiotemporal Effects}

We may also consider stochastic effects (linear and nonlinear critical dynamics) for systems far from equilibrium using the RG generator (1) or (2). Generally, when the 
stochastic effects due to the time-varying noises on the ordering and symmetry breakings need to be considered for a non-equilibrium dynamical system, the conjugate ("momentum") fields of the ordering ("coordinate") fields due to the imposed noise must be generated and included in the scale-dependent stochastic effective action. Space prevents us from delving into the complicated details of such calculations. For readers who are interested in such details, they are invited to peruse the discussions given in $[4,8]$.

Instead, we consider the results of a simple example below to demonstrate the typical spatiotemporal effects in gravitational evolution. Consider, for example, the case of $\varphi$ Lifshitz helical order-breaking with weak mode coupling and its dynamics driven by a stochastic noise characterized by an isotropic propagator $i\left(k^{\sigma}+r+i \omega / \Gamma_{k}\right)$ where $\sigma=2 L$ with $L$ being the Lifshitz character, $\omega$ the Fourier component of the time variable, and $\Gamma_{k} \propto k^{y}$ the characteristics of the noise [4]. We may then append the ordering field $\varphi$ with its conjugate field $\pi$ according to [4] and search for the perturbed nontrivial fixed points following the same procedure as that was employed to obtain the results given in Eqs. $(4,5)$.

If we wish to extend the calculations to second order effects in $\varepsilon_{O}$ in the perturbation scheme, then we must use the full functional form of (2). The propagator matrix corresponding to this model is given below:

$$
\left(\begin{array}{cc}
0 & i\left(k^{\sigma}+r+i \omega / \Gamma_{k}\right) \\
i\left(k^{\sigma}+r-i \omega / \Gamma_{k}\right) & \left(2 \Gamma_{h}\right)^{-1}
\end{array}\right)
$$

Since it may be shown that the (1,1)-matrix element of (6) will not couple into the calculations of the quantities of interest, we have conveniently set it equal to 0 . The 
eigenfunctions in functional space are now eigen-operators. Such an analysis has been performed explicitly by Vvedensky and Chang in detail in [15] for the $k^{2}$-propagator (i.e., for $\sigma=2$ ). Extension of the results to the isotropic Lifshitz propagator matrix (6) may easily be accomplished by replacing the critical propagator exponent " 2 " by $\sigma$. The results of these calculations can, of course, also be calculated directly using the differential equations obtained from the generators [4].

Based on the results given in [4], we find by setting $n=1$ the nontrivial fixed points for the gravitational potential $\varphi$ for $d<2 \sigma$ and $y \neq 0$ to first order in $\varepsilon \equiv 2 \sigma-d$ with perturbed eigenvalues the same as those of the isotropic propagator version of $(4,5)$ to first order and an additional dynamic exponent $z=\sigma+y-\eta$ (which measures the anisotropic scaling between time and space) where the correlation function exponent $\eta$ for $\sigma=2 L$ when $L$ is an integer is:

$$
\eta=\varepsilon^{2}\left[(-1)^{L+1} \Gamma^{2}(2 L) / 27 L \Gamma(L) \Gamma(3 L)\right]+O\left(\varepsilon^{3}\right)
$$

and $z=\sigma+y$ when $L$ is not an integer.

We recall that in the earlier first order calculations, the correlation function exponent (anomalous dimension) was assumed to be of higher order in the perturbation expansion. The result in (7) confirms such an assumption.

When $y=0$, on the other hand, the dynamic exponent for our present analysis is found to be $z=\sigma-\eta+I$ with

$$
I=\varepsilon^{2} 2^{\sigma} \Gamma(\sigma) / 27 \int_{0}^{\infty} d t\left[\int_{0}^{\infty} d x \exp \left(-t x^{\sigma}\right) J_{\sigma-1}(x)\right]^{3}+O\left(\varepsilon^{3}\right)
$$

We note from this example that orderings and symmetry breakings are generally anisotropic between space and time in dynamical evolution due to classical 
spatiotemporal fluctuations. Because of the sporadic localized variations of $\sigma_{i}$, the antiscreening/screening effects and appearance of virtual particles may be characterized in terms of spatiotemporal varying (and possibly singular) geometries (a hallmark of true dynamical complexity).

Even for situations of phase transitions involving uniformly or commensurately ordered states or for isotropic Lifshitz transitions that led to the result given in (8), there can be antiscreening/screening effects due to the second order effects in $\varepsilon_{0}$, which can take on logarithmic scaling characteristics.

\section{Interpretation}

In the above fixed point analyses, we found that critical RG trajectories may accompany cusp-like singularities of the coarse-grained scale-running gravitational constant in momentum space. Actual RG evolutions of the dynamic medium, on the other hand, do not need to follow such trajectories exactly. In fact, they generally are continuous paths that may evolve close to or away from the critical trajectories. The antiscreening/screening effects then vary up and down (increase and decrease) following these trajectories. If one follows such trajectories and integrate the RG functional integrodifferential equation (1), we obtain the full effective action (Helmholtz free energy) $A(\bar{\psi})$ of the local evolving medium $[8,16,17]$. Thus, nonlinear equation(s) of state, which may be obtained from the integrated effective action of the evolving medium, cannot be assumed a priori because they change sporadically and locally from criticality to criticality and within the crossover domains depending on its complexity structure. Since the RG generator is nonlinear and singular, generally the integrated effective actions or Helmholtz free energies are singular functions of the global nonlinear 
invariants (in the form of nonlinear functions of the coupling constants). It is within the crossover regions (transition regions of singular behavior) among the admissible fixed points that the apparent coarse-grained non-Newtonian effects due to the nonlinear running of the gravitational constant that produce the complexity behavior that we have been addressing in this example. Due to the influence of the inherent classical fluctuations in dynamical evolution, CILOMAS leads the stochastic system to exhibit the fading-in and fading-out antiscreening/screening (including dark matter) effect of symmetry breakings of tangled helical orderings induced by the coarse-grained scalerunning of the propagator-coupling constant (the gravitational constant in our example).

Analogous examples may be found in various condensed matters [8.16.17].

\section{Multifractals}

An alternative point of view of the complex medium is that at each local state of the full RG space, the medium may be considered to be characterized by a local invariant (e.g., that of the coupling constants including $G$ ) with a fractal singularity (power law scaling with generally an irrational exponent) which varies from one locality to another according to the change of the RG parameters and therefore the local state.

This is the point of view taken by the recently developed concept of rank-ordered multifractal analysis (ROMA) [10,11]. This technique introduced by Chang and Wu [18] determines explicitly the complexity of the medium in terms of an implicit spectrum of fractals (i.e., multifractals) with the local fractal dimension "s" expressed as a nonlinear function of the local invariant " $Y$ ". Such type of analyses contains much more statistical information for comparison than those provided by the conventional spectrum density and correlation function techniques. 


\section{Summary and Concluding Remarks}

In summary, we have demonstrated analytically that fixed points are abundant in the RG phase space that can provide the onset of symmetry breakings and crossovers of competing helically ordered states with fractal variations of the propagator-coupling constants such as the gravitational constant during dynamical evolution under general spatiotemporal fluctuations and self-organization. For the example discussed, the analysis is based on the scale-dependent effective action for the Newtonian approximation and classical complexity phenomena with the running of the propagatorcoupling constant $G$. The full effect of such complex phase-transition behavior indicates that phenomena of sporadic and localized antiscreening/screening and their collaborative effects with the orderings and competitions of orderings of self-organized and generally tangled helical coherent structures exist during general dynamic evolution with the development of large structures and multifractal fluctuations accompanied by coarsegrained apparent virtual-particle effects such as dark matters. Such complex and multifractal behavior can be analyzed and compared utilizing refined statistical methods such as the recently developed rank-ordered multifractal analyses (ROMA) $[10,18]$ based

on both the results of realistic large scale numerical simulations with a running propagator-coupling constant and actual observations.

These ideas can easily be applied to a variety of condensed matter systems exhibiting the novel phenomenon of CILOMAS, where the coupling constant(s) of the propagator(s) is (are) scale dependent.

\section{Acknowledgment}

This research was partially supported by a grant from NSF of the US government. 


\section{References}

[1] R.M. Hornreich, M. Luban, and S. Shtrikman, Phys. Rev. Lett. 35, (1975) 1678.

[2] J.F. Nicoll, G.F. Tuthill, T.S. Chang, and H.E. Stanley, Phys. Lett. A, 58 (1976) 1.

[3] J. F. Nicoll and T.S. Chang, Phys. Lett. A 62, (1977) 287.

[4] T.S. Chang, J.F. Nicoll, and J.E. Young, Phys. Lett. A 67 (1978) 287.

[5] K.G. Wilson and J. Kogut, Phys. Rep. 12C (1974) 75.

[6] K.G. Wilson, Rev. Mod. Phys., 55 (1983) 583.

[7] F. Wegner, Critical states, general aspects, in: C. Domb and M.S. Green (Eds.),

Phase Transitions and Critical Phenomena, vol. 6 (Ch.2), p. 7, Academic Press, London, 1976.

[8] T.S. Chang, D.D. Vvedensky, and J.F. Nicoll, Phys. Rep. 217 (1992) 279.

[9] F. Wegner and A. Houghton, Phys. Rev. A 8 (1973) 401.

[10] T.T.S. Chang, An Introduction to Space Plasma Complexity, Cambridge University Press, New York, 2015.

[11] T. Chang, C.C. Wu, M. Echim, H. Lamy, M. Vogelsberger, L. Hernquist, and D.

Sijacki, Pure Appl. Geophys., 171 (2014) 1089.

[12] C.C. Wu and T. Chang, Nonlin. Processes Geophys. 18 (2011) 261.

[13] J.V. Narlikar, Introduction to Cosmology, second edition (Cambridge University Press, Cambridge, 1993).

[14] J.F. Nicoll, T.S. Chang, and H.E. Stanley, Phys. Rev. Lett., 33 (1974) 540.

[15] D.D. Vvedensky and T.S. Chang, Phys. Lett. A, 90 (1982) 459.

[16] J.F. Nicoll, T.S. Chang, and H.E. Stanley, Phys. Rev. Lett., 32 (1974) 1446.

[17] J.F. Nicoll and T.S. Chang, Phys. Rev. A, 17 (1978) 2083. 
[18] T. Chang and C.C. Wu, Phys. Rev. E, 77 (2008) 045401(R). 